\title{
Pacientes em centro de referência para Hanseníase: Rio de Janeiro e Duque de Caxias, 1986-2008
}

\author{
Patients from a Reference Center for Leprosy: \\ Rio de Janeiro and Duque de Caxias, 1986-2008
}

Mariana de Andrea Vilas Boas Hacker ${ }^{1}$

Anna Maria Sales ${ }^{1}$

Edson Cláudio Araripe Albuquerque ${ }^{1}$

Emanuel Rangel ${ }^{1}$

Jose Augusto Costa Nery ${ }^{1}$

Nadia Cristina Duppre ${ }^{1}$

Euzenir Nunes Sarno ${ }^{1}$

${ }^{1}$ Fundação Oswaldo Cruz.

Abstract The scope of this study was to compare epidemiological data on leprosy patients living in two cities with different socioeconomic and endemic profiles that were monitored in a single center of reference. A descriptive study was made of data from patients in the Souza Araújo Outpatient facility treated in the period 1986-2008, who were resident in the cities of Rio de Janeiro $=1353$ ) and Duque de Caxias $(n=336)$. Results: Among patients from Duque de Caxias, in comparison with patients from Rio de Janeiro, there was a higher proportion of cases: below the age of 15 years, multibacillary, higher initial bacilloscopic index (BI) and cases detected through surveillance of contacts. Patients in Duque de Caxias had lower average incomes and education levels. There were no statistically significant differences regarding gender, disability level, reaction in the diagnosis, final BI, bandonment and regularity of treatment. The differences found between the patients monitored in a single center of reference, could be partly related to contextual differences between the municipalities. On the other hand, it was observed that the provision of treatment and monitoring can minimize the effect of different contextual factors on health outcomes.

Key words Leprosy, Epidemiology, Brazil.
Resumo O objetivo deste trabalho foi comparar dados epidemiológicos de pacientes de hanseníase residentes em dois municipios com perfil socioeconômico e nível de endemicidade diferentes e que foram acompanhados em um mesmo centro de referência. Foi realizado um estudo descritivo dos dados de pacientes tratados no ambulatório Souza Araújo, 1986 a 2008, residentes nos municípios do Rio de Janeiro $(n=1353)$ e Duque de Caxias ( $n=$ 336). Entre os pacientes desta cidade, em comparação com os da outra, observou-se maior proporção de casos: com idade inferior a 15 anos, multibalicares, com maior indice baciloscópico (IB) inicial, e detectados através da vigilância de contatos. Os pacientes de Duque de Caxias apresentaram menor renda média e nível de escolaridade. Não foram observadas diferenças estatisticamente significativas quanto ao sexo, grau de incapacidade inicial, reação no diagnóstico, IB final, abandono e regularidade do tratamento. As diferenças encontradas entre os pacientes acompanhados em um mesmo centro de referência poderiam estar, em parte, relacionadas a diferenças contextuais existentes entre os municípios. Por outro lado, observou-se que a oferta de tratamento e acompanhamento podem minimizar o efeito que os fatores contextuais apresentam sobre os desfechos de saúde.

Palavras-chave Hanseniase, Epidemiologia, Brasil

Manguinhos. 21045-900

Rio de Janeiro RJ.

marianah@ioc.fiocruz.br 


\section{Introdução}

A Hanseníase, ainda constitui um relevante problema de saúde publica apesar dos esforços para sua eliminação, com o estabelecimento de metas e estratégias definidas pela Organização Mundial de Saúde. O país é o segundo no mundo com maior número de casos novos detectados, precedido apenas pela Índia ${ }^{1}$.

A detecção anual vem declinando, em 2003 foram mundialmente detectados mais de $514 \mathrm{mil}$ casos e em 2009 em torno de 244 mil casos. No inicio de 2010, a prevalência global de hanseníase era de cerca de 212 mil casos. No Brasil, em 2009, foram detectados 37.610 novos casos ${ }^{1}$.

Observa-se uma tendência de estabilização dos coeficientes de detecção no Brasil, porém estes apresentam variação nas diferentes regiões. Embora alguns municípios já tenham alcançado a meta de eliminação da doença, as regiões Norte, Centro-Oeste e Nordeste ainda apresentam altos patamares de casos da doença ${ }^{2}$.

A epidemiologia dos fatores de risco individuais tem sido o paradigma dominante nas últimas décadas, com foco nos comportamentos e exposições individuais. Nos últimos anos, porém, a epidemiologia social, área da epidemiologia que estuda a distribuição social e os determinantes sociais de saúde, tem-se tornado proeminente ${ }^{3,4}$.

Conforme discutido por Cunha et al. ${ }^{5}$, o estado do Rio de Janeiro ainda apresenta um cenário desfavorável, devendo-se ponderar fatores como densidade populacional, hábitos de vida, aspectos culturais e condições sanitárias e de moradia.

Segundo dados preliminares de 2008, o coeficiente de detecção no estado do Rio de Janeiro foi de 11,84 por 100 mil habitantes. O coeficiente de detecção em menores de 15 anos, nos municípios do Rio de Janeiro e Duque de Caxias demonstrou serem áreas de alta e média endemicidade para a hanseníase, respectivamente ${ }^{6}$. Nesses municípios estão concentrados o maior número de casos de hanseníase em todo o estado. A capital de estado, apresenta indicadores municipais mais favoráveis em relação à saúde, à habitação e à renda do que os demais municípios da região metropolitana geograficamente localizados na baixada fluminense . $^{7}$

Segundo dados do censo do ano 2000, no município do Rio de Janeiro, a proporção de pessoas que vivem em domicílios com água encanada era de $97,80 \%$, e a proporção de pessoas que vivem em domicílio com densidade acima de 2 pessoas por dormitório era de $17,14 \%$. No mu- nicípio de Duque de Caxias esses indicadores foram iguais $87,27 \%$, e $27,24 \%$, respectivamente. O índice de desenvolvimento humano (IDH), para o município do Rio de Janeiro foi de 0,842 e para o município de Duque de Caxias foi de $0,753^{7}$.

O objetivo do estudo foi comparar as características clínicas e epidemiológicas de pacientes acompanhados em um Centro de Referência Nacional de Hanseníase e residentes em dois Municípios com diferentes níveis de endemicidade.

\section{Métodos}

A clientela do Ambulatório Souza Araújo é composta por indivíduos da região metropolitana do Rio de Janeiro, além de municípios do interior do estado. A demanda de pacientes atendidos é proveniente de encaminhamentos realizados por quaisquer serviços de saúde (públicos ou privados), procura espontânea ou de vigilância de contatos. Após a confirmação clínica, histopatológica e bacteriológica da hanseníase os pacientes são tratados e acompanhados.

O Ambulatório Souza Araújo (ASA), do Laboratório de Hanseníase da Fiocruz, Centro de Referencia Nacional para Hanseníase oferece uma assistência diferenciada à população, contando, além do serviço especializado de dermatologia, serviços na área de neurologia, com a realização de exames especializados para diagnóstico diferencial, fisioterapia para tratamento e prevenção de incapacidades, e assistência socioeducativa, que inclui iniciativas de educação em saúde para pacientes e contatos, bem como a vigilância epidemiológica destes.

As informações dos pacientes do ASA são registradas em banco de dados contendo informações socioeconômicas e parâmetros clínicolaboratoriais dos pacientes. A utilização desses dados para publicação é aprovada pelo comitê de ética em pesquisa da Fundação Oswaldo Cruz, em acordo com a Declaração de Helsinque.

Para avaliar as características clínicas e epidemiológicas de pacientes provenientes de áreas com diferentes níveis de endemicidade foram incluídos neste estudo apenas os pacientes residentes nos municípios do Rio de Janeiro (alta endemicidade) e de Duque de Caxias (média endemicidade), atendidos no ASA entre os anos de 1986 a 2008, utilizando-se o banco de dados deste. Nesse período, 52\% (1353) dos pacientes eram residentes no Município do Rio de Janeiro e 12,9 \% (336) em Duque de Caxias, este último representando o município de residência responsável 
pela segunda maior demanda dos pacientes atendidos no ASA.

As características socioeconômicas, clínicas e epidemiológicas foram analisadas segundo o município de residência e foram comparadas em três períodos: 1986 a 1991, 1992 a 1998 e 1999 a 2008.

Foram comparadas as distribuições por sexo, idade e escolaridade (anos completos de estudo), a média da renda familiar (em salários mínimos), modo de detecção (espontânea, encaminhamento-serviço público, encaminhamentoserviço particular, vigilância de contatos), classificação operacional (multibacilar-MB, paucibacilar-PB), grau de incapacidade ( 0, I, II) no início e ao final do tratamento, índice baciloscópico no início e ao final do tratamento, presença de quadro reacional no diagnóstico, abandono de tratamento e regularidade do esquema de tratamento poliquimioterápico (para paucibacilares 6 doses em até 9 meses e para multibacilares 12 doses em até 18 meses e para multibacilares com esquema anterior de 24 doses em até 36). Para avaliar a significância estatística foi utilizado teste qui-quadrado e teste-t de Student na análise bivariada. Foi realizada análise multivariada para cada período analisado utilizando a regressão logística. Foram construídos 3 modelos, um para cada período, utilizando o método forward para seleção das variáveis. Foi considerado nível de significância de $10 \%$ para entradas das variáveis e $15 \%$ para a retirada no modelo.

\section{Resultados}

Durante o período do estudo foram registrados 2.608 pacientes no ASA, destes 1.353 (52\%) pacientes eram provenientes de encaminhamentos do Município do Rio de Janeiro e 336 (12,9\%) do de Duque de Caxias.

A análise bivariada (Tabelas 1 e 2) permitiu identificar as características epidemiológicas que se apresentaram estatisticamente diferentes entre os grupos de pacientes residentes nos municípios do Rio de Janeiro e Duque de Caxias atendidos em um Centro Nacional de Referência para Hanseníase.

A Tabela 1 apresenta os resultados da análise bivariada em relação a características socioeconômicas dos pacientes avaliados. Em relação ao sexo, não foram observadas diferenças estatisticamente significativas entre os pacientes provenientes do município de Duque de Caxias e os do Rio de Janeiro, observou-se apenas uma peque- na predominância de pacientes do sexo masculino em ambos.

Entre os pacientes provenientes do município de Duque de Caxias, observou-se uma maior proporção de casos com idade inferior a 15 anos quando comparados aos pacientes residentes no município do Rio de Janeiro em todos os períodos, especialmente no último período (14,5 e $7,4 \%$, respectivamente) quando essa diferença foi estatisticamente significativa ( $\mathrm{p}$-valor $=0,013$ ).

Os pacientes residentes no município de $\mathrm{Du}$ que de Caxias apresentaram menor nível de escolaridade e renda familiar média inferior quando comparados aos pacientes residentes no município do Rio de Janeiro, essas diferenças foram estatisticamente significativas.

Em relação à renda familiar, no primeiro período, os pacientes de ambos os municípios apresentaram médias similares (4,67 e 4,26, p-valor $=0,829$ ), no entanto, nos dois últimos períodos, observa-se uma redução da renda média familiar entre os pacientes de Duque de Caxias, tornando as diferenças estatisticamente significativas em comparação com os pacientes do Rio de Janeiro: no segundo período foi de 2,84 versus $5,11$ ( $\mathrm{p}$-valor $=0,006)$ respectivamente e no terceiro período foi de 2,74 versus 4,57 ( $\mathrm{p}$-valor $=$ 0,012 ) respectivamente.

Observou-se que a maioria dos pacientes foi detectada através de encaminhamentos dos serviços públicos tanto no grupo de pacientes do município do Rio de Janeiro quanto de Duque de Caxias. Observa-se maior proporção de casos de hanseníase detectados pelo serviço, através da vigilância de contatos entre os pacientes residentes em Duque de Caxias em todos os períodos. No período de 1897 a 1991, essa proporção foi igual a 20,6\% entre os pacientes residentes em Duque de Caxias e igual a 11,8\% entre os pacientes residentes no município do Rio de Janeiro. No período de 1992 a 1998 essas proporções foram de $26,5 \%$ e $11,4 \%$, respectivamente e no período de 1999 a 2008, foram de 29,1\% e 12,3\%, respectivamente. As diferenças foram estatisticamente significativas em todos os períodos. A Tabela 2 apresenta a análise bivariada em relação a características clínicas dos pacientes analisados. Observa-se maior proporção de casos MB entre os pacientes provenientes do município de $\mathrm{Du}$ que de Caxias quando comparados àqueles residentes no do Rio de Janeiro. Essa diferença foi estatisticamente significativa $(\mathrm{p}$-valor $=0,044)$ no período de 1992 a 1998, quando as proporções foram de $55,6 \%$ e $44,7 \%$, respectivamente. No período mais recente, observa-se uma redução 
nessas proporções para ambos os grupos de pacientes, sem diferença estatística entre eles (passando para $47,0 \%$ entre os pacientes de Duque de Caxias e $42,7 \%$ entre os pacientes do município do Rio de Janeiro).
No período de 1992 a 1998, a frequência de presença de quadro reacional no momento do diagnóstico foi mais elevada entre o grupo de pacientes residentes em Duque de Caxias $(47,9 \%)$, do que entre o grupo de pacientes resi-

Tabela 1. Análise bivariada. Características socioeconômicas dos pacientes do ASA. Duque de Caxias e Rio de Janeiro, 1986-2008.

\begin{tabular}{|c|c|c|c|c|c|c|}
\hline \multirow[b]{2}{*}{$\begin{array}{l}\text { Variáveis } \\
\text { socioeconômicas }\end{array}$} & \multicolumn{3}{|c|}{1986 a 1991} & \multicolumn{3}{|c|}{1992 a 1998} \\
\hline & $\begin{array}{l}\text { Duque de Caxias } \\
\qquad \mathrm{n}=102\end{array}$ & $\begin{array}{c}\text { Rio de Janeiro } \\
\quad \mathrm{n}=490\end{array}$ & p-valor & $\begin{array}{l}\text { Duque de Caxias } \\
\qquad \mathrm{n}=117\end{array}$ & $\begin{array}{l}\text { Rio de Janeiro } \\
\quad \mathrm{n}=334\end{array}$ & p-valor \\
\hline \multicolumn{7}{|l|}{ Sexo } \\
\hline Feminino & 41,2 & 43,7 & 0,643 & 45,3 & 44,0 & 0,809 \\
\hline Masculino & 58,8 & 56,3 & & 54,7 & 56,0 & \\
\hline \multicolumn{7}{|l|}{ Idade } \\
\hline$<15$ anos & 12,7 & 10,8 & 0,573 & 17,1 & 12,0 & 0,164 \\
\hline$\geq 15$ anos & 87,3 & 89,2 & & 82,9 & 88,0 & \\
\hline \multicolumn{7}{|l|}{ Escolaridade ${ }^{*}$} \\
\hline Até 7 anos & 64,5 & 74,1 & 0,574 & 72,3 & 60,8 & 0,025 \\
\hline Entre 8 e 10 anos & 22,6 & 15,7 & & 21,7 & 20,8 & \\
\hline Acima de 10 anos & 12,9 & 10,2 & & 6,0 & 18,4 & \\
\hline \multicolumn{7}{|l|}{ Modo de Detecção ${ }^{* *}$} \\
\hline Espontânea & 7,8 & 5,7 & 0,096 & 5,6 & 8,1 & 0,009 \\
\hline Encaminhamento & 8,8 & 13,9 & & 13,7 & 21,0 & \\
\hline Médico Particular & & & & & & \\
\hline Encaminhamento & 47,1 & 53,7 & & 51,3 & 55,1 & \\
\hline Serviço Público & & & & & & \\
\hline Vigilância de contatos & 20,6 & 11,8 & & 26,5 & 11,4 & \\
\hline Renda Familiar $^{* * *}$ & 4,67 & 4,26 & 0,829 & 2,84 & 5,11 & 0,006 \\
\hline \multirow[t]{2}{*}{ Média (desvio- padrão) } & $(7,51)$ & $(4,38)$ & & $(2,51)$ & $(6,89)$ & \\
\hline & & 99 a 2008 & & & Total & \\
\hline $\begin{array}{c}\text { Variáveis } \\
\text { socioeconômicas }\end{array}$ & $\begin{array}{l}\text { Duque de Caxias } \\
\qquad \mathbf{n}=117\end{array}$ & $\begin{array}{c}\text { Rio de Janeiro } \\
\quad n=529\end{array}$ & p-valor & $\begin{array}{l}\text { Duque de Caxias } \\
\qquad \mathrm{n}=336\end{array}$ & $\begin{array}{l}\text { Rio de Janeiro } \\
\quad n=1353\end{array}$ & p-valor \\
\hline \multicolumn{7}{|l|}{ Sexo } \\
\hline Feminino & 41,0 & 42,2 & 0,823 & 42,6 & 43,2 & 0,854 \\
\hline Masculino & 59,0 & 57,8 & & 57,4 & 56,8 & \\
\hline \multicolumn{7}{|l|}{ Idade } \\
\hline$<15$ anos & 14,5 & 7,4 & 0,013 & 14,9 & 9,8 & 0,009 \\
\hline$\geq 15$ anos & 85,5 & 92,6 & & 85,1 & 90,2 & \\
\hline \multicolumn{7}{|l|}{ Escolaridade ${ }^{*}$} \\
\hline Até 7 anos & 67,5 & 61,9 & 0,164 & 68,9 & 63,2 & 0,013 \\
\hline Entre 8 e 10 anos & 20,2 & 18,2 & & 21,1 & 18,5 & \\
\hline Acima de 10 anos & 12,3 & 19,9 & & 10,1 & 18,3 & \\
\hline \multicolumn{7}{|l|}{ Modo de Detecção* } \\
\hline Espontânea & 6,8 & 5,7 & $<0,001$ & 6,5 & 6,3 & $<0,001$ \\
\hline $\begin{array}{l}\text { Encaminhamento } \\
\text { Médico Particular }\end{array}$ & 11,1 & 17,2 & & 11,3 & 16,9 & \\
\hline $\begin{array}{l}\text { Encaminhamento } \\
\text { Serviço Público }\end{array}$ & 45,3 & 61,2 & & 47,9 & 55,0 & \\
\hline Vigilância de contatos & 29,1 & 12,3 & & 25,6 & 11,9 & \\
\hline Renda Familiar $^{* * *}$ & 2,74 & 4,57 & 0,012 & 2,96 & 4,07 & 0,007 \\
\hline Média (desvio- padrão) & $(0,264)$ & $(0,213)$ & & $(3,37)$ & $(5,32)$ & \\
\hline
\end{tabular}

‘anos completos de estudo, ${ }^{* *}$ demais modos de detecção foram classificados como 'outros', ${ }^{* * *}$ em salários mínimos. 
Tabela 2. Análise bivariada. Características clínicas dos pacientes do ASA. Duque de Caxias e Rio de Janeiro, 1986-2008.

\begin{tabular}{|c|c|c|c|c|c|c|}
\hline \multirow[b]{2}{*}{ Variáveis clínicas } & \multicolumn{3}{|c|}{1986 a 1991} & \multicolumn{3}{|c|}{1992 a 1998} \\
\hline & $\begin{array}{c}\text { Duque de } \\
\text { Caxias }\end{array}$ & $\begin{array}{l}\text { Rio de } \\
\text { Janeiro }\end{array}$ & p-valor & $\begin{array}{c}\text { Duque de } \\
\text { Caxias }\end{array}$ & $\begin{array}{l}\text { Rio de } \\
\text { Janeiro }\end{array}$ & p-valor \\
\hline \multicolumn{7}{|l|}{ Classificação Operacional } \\
\hline $\mathrm{MB}$ & 50,5 & 46,1 & 0,420 & 55,6 & 44,7 & 0,044 \\
\hline $\mathrm{PB}$ & 49,5 & 53,9 & & 44,4 & 55,3 & \\
\hline Reação & 15,7 & 17,6 & 0,650 & 47,9 & 35,6 & 0,019 \\
\hline \multicolumn{7}{|l|}{ GI inicial } \\
\hline 0 & 57,0 & 60,3 & 0,434 & 69,3 & 72,5 & 0,287 \\
\hline I & 26,9 & 21,0 & & 19,3 & 13,5 & \\
\hline II & 16,1 & 18,8 & & 11,4 & 14,1 & \\
\hline \multicolumn{7}{|l|}{ GI final } \\
\hline 0 & 64,2 & 67,7 & 0,709 & 67,1 & 74,4 & 0,451 \\
\hline $\mathrm{I}$ & 19,8 & 19,2 & & 13,8 & 12,3 & \\
\hline II & 16,0 & 12,7 & & 18,1 & 13,4 & \\
\hline Regularidade no tratamento & 82,4 & 86,5 & 0,309 & 85,8 & 88,6 & 0,451 \\
\hline Abandono de tratamento & 5,9 & 5,3 & 0,815 & 4,3 & 5,1 & 0,724 \\
\hline \multicolumn{7}{|l|}{ IB inicial } \\
\hline 0 & 53,8 & 59,0 & 0,005 & 47,4 & 58,1 & 0,013 \\
\hline $0<\mathrm{IB} \leq 3$ & 21,5 & 28,9 & & 31,6 & 24,3 & \\
\hline IB $>3$ & 24,7 & 12,1 & & 21,1 & 17,6 & \\
\hline \multicolumn{7}{|l|}{ IB final } \\
\hline 0 & 49,1 & 50,6 & 0,980 & 37,7 & 50,3 & 0,100 \\
\hline $0<\mathrm{IB}<3$ & 43,4 & 41,2 & & 55,1 & 39,8 & \\
\hline \multirow[t]{2}{*}{$\mathrm{IB}>3$} & 7,5 & 8,2 & & 7,2 & 9,9 & \\
\hline & \multicolumn{3}{|c|}{1999 a 2008} & \multicolumn{3}{|c|}{ Total } \\
\hline Variáveis clínicas & $\begin{array}{c}\text { Duque de } \\
\text { Caxias }\end{array}$ & $\begin{array}{c}\text { Rio de } \\
\text { Janeiro }\end{array}$ & p-valor & $\begin{array}{c}\text { Duque de } \\
\text { Caxias }\end{array}$ & $\begin{array}{c}\text { Rio de } \\
\text { Janeiro }\end{array}$ & p-valor \\
\hline \multicolumn{7}{|l|}{ Classificação Operacional } \\
\hline $\mathrm{MB}$ & 47,0 & 42,7 & 0,397 & 51,0 & 44,4 & 0,035 \\
\hline PB & 53,0 & 57,3 & & 49,0 & 55,6 & \\
\hline Reação & 16,2 & 23,1 & 0,106 & 27,1 & 24,2 & 0,300 \\
\hline \multicolumn{7}{|l|}{ GI inicial } \\
\hline 0 & 65,2 & 64,7 & 0,578 & 64,3 & 65,1 & 0,116 \\
\hline I & 20,0 & 17,1 & & 21,7 & 17,5 & \\
\hline II & 14,8 & 18,2 & & 14,0 & 17,4 & \\
\hline \multicolumn{7}{|l|}{ GI final } \\
\hline 0 & 66,7 & 68,8 & 0,752 & 66,4 & 69,9 & 0,267 \\
\hline I & 18,4 & 19,3 & & 17,2 & 17,5 & \\
\hline II & 14,9 & 12,0 & & 16,4 & 12,6 & \\
\hline Regularidade no tratamento & 95,4 & 93,3 & 0,420 & 88,2 & 89,7 & 0,436 \\
\hline Abandono de tratamento & 0,9 & 4,3 & 0,100 & 3,6 & 4,9 & 0,381 \\
\hline \multicolumn{7}{|l|}{ IB inicial } \\
\hline 0 & 49,5 & 57,0 & 0,272 & 50,0 & 58,0 & 0,006 \\
\hline $0<\mathrm{IB} \leq 3$ & 24,3 & 22,9 & & 26,1 & 25,5 & \\
\hline $\mathrm{IB}>3$ & 26,1 & 20,0 & & 23,9 & 16,7 & \\
\hline \multicolumn{7}{|l|}{ IB final } \\
\hline 0 & 45,5 & 35,9 & 0,478 & 43,4 & 45,9 & 0,491 \\
\hline $0<\mathrm{IB}<3$ & 31,8 & 39,8 & & 45,2 & 40,3 & \\
\hline $\mathrm{IB}>3$ & 22,7 & 24,3 & & 11,4 & 13,7 & \\
\hline
\end{tabular}


dentes no Rio de Janeiro (35,6\%), quando essas diferenças foram estatisticamente significativas ( $\mathrm{p}$-valor $=0,019)$.

Em relação ao grau de incapacidade física no início e no final do tratamento e à regularidade no tratamento, não foram observadas diferenças estatisticamente significativas entre os dois grupos de pacientes. Observa-se que no último período avaliado, a regularidade no tratamento (95,4 e 93,3\% entre pacientes de Duque de Caxias e Rio de Janeiro, respectivamente) foi mais elevada do que nos período anteriores.

Observou-se uma redução na proporção de abandono de tratamento ao longo do tempo, entre os pacientes residentes em ambos os municípios, no entanto no último período a proporção de abandono foi significativamente menor entre os pacientes residentes em Duque de Caxias $(0,9 \%$ versus $4,3 \%, \mathrm{p}=0,100)$ respectivamente.

Quanto ao IB inicial, observa-se diferenças estatisticamente significativas, principalmente nos 2 primeiros períodos do estudo, entre pacientes com IB acima de 3 residentes de Duque de Caxias, quando comparados àqueles residentes no município do Rio de Janeiro. Com relação ao IB final foram observadas diferenças estatisticamente significativas no período de 1992 a 1998, com predominância de IB igual a zero entre os pacientes do Rio de Janeiro e IB inferior ou igual a 3 entre os pacientes de Duque de Caxias, e a proporção de pacientes com IB superior a 3, maior entre os pacientes do Rio de Janeiro $(\mathrm{p}=0,100)$.

Uma vez que as características observadas apresentam relação entre si, a análise multivariada permitiu identificar quais características epidemiológicas foram de fato responsáveis pelas diferenças observadas entre os dois grupos de pacientes. Na regressão logística (Tabela 3), quando consideradas todas as variáveis conjuntamente, o modo de detecção, o IB inicial e a regularidade no tratamento se mostraram estatisticamente significativas no primeiro período (modelo 1); no segundo período (modelo 2), mostraram-se significativas as variáveis idade, reação no diag-

Tabela 3. Análise multivariada. Características socioeconômicas e clínicas dos pacientes do ASA. Duque de Caxias e Rio de Janeiro, 1986-2008.

\begin{tabular}{|c|c|c|c|c|c|c|c|c|c|}
\hline \multirow[b]{3}{*}{ Variáveis } & \multicolumn{3}{|c|}{ Modelo 1} & \multicolumn{3}{|c|}{ Modelo 2} & \multicolumn{3}{|c|}{ Modelo 3} \\
\hline & \multicolumn{3}{|c|}{1986 a 1991} & \multicolumn{3}{|c|}{1992 a 1998} & \multicolumn{3}{|c|}{1999 a 2008} \\
\hline & Coeficiente & EP & p-valor & Coeficiente & EP & p-valor & Coeficiente & EP & p-valor \\
\hline Idade & & & & & & 0,072 & & & 0,008 \\
\hline$<15$ anos & & & & - & & & - & & \\
\hline$\geq 15$ anos & & & & 1,179 & 0,656 & & 2,598 & 0,981 & \\
\hline Modo de Detecção* & & & 0,065 & & & & & & 0,048 \\
\hline Espontânea & $-0,889$ & 1,395 & & & & & 0,289 & 0,992 & \\
\hline Encaminhamento & 0,404 & 1,285 & & & & & 1,033 & 0,956 & \\
\hline Médico Particular & & & & & & & & & \\
\hline Encaminhamento & $-4,464$ & 2,172 & & & & & $-0,603$ & 1,031 & \\
\hline Serviço Público & & & & & & & & & \\
\hline Vigilância de contatos & $-2,591$ & 1,962 & & & & & $-0,236$ & 1,135 & \\
\hline Reação & & & & $-0,902$ & 0,452 & 0,046 & 0,937 & 0,454 & 0,039 \\
\hline Regularidade no tratamento & 4,081 & 1,830 & 0,026 & & & & & & \\
\hline IB inicial & & & 0,019 & & & & & & \\
\hline 0 & - & & & & & & & & \\
\hline $0<\mathrm{IB} \leq 3$ & 1,725 & 1,925 & & & & & & & \\
\hline IB $>3$ & $-1,925$ & 1,740 & & & & & & & \\
\hline GI final & & & & & & & & & 0,113 \\
\hline 0 & & & & & & & - & & \\
\hline I & & & & & & & $-0,057$ & 0,486 & \\
\hline II & & & & & & & $-1,189$ & 0,584 & \\
\hline Renda Familiar & & & & 0,141 & 0,068 & 0,037 & & & \\
\hline
\end{tabular}

* categoria 'outro' foi usada como referência. $\mathrm{EP}=$ erro-padrão 
nóstico e renda familiar, e no terceiro período (modelo 3), as variáveis idade, modo de detecção, reação no diagnóstico e grau de incapacidade final mostraram-se significativas.

\section{Discussão}

Na casuística de pacientes do ASA, o grupo de pacientes residentes em Duque de Caxias apresentou maior proporção de casos com idade inferior a 15 anos, maior proporção de pacientes com IB inicial elevado, maior proporção de casos detectados através da vigilância de contatos, maior proporção de casos com incapacidades ao final do tratamento e menor renda média familiar.

Embora tenha sido registrada uma redução importante na detecção desses casos no país $(7,98$ em 2003 para 5,89 por 100 mil em 2008) ${ }^{8}$, segundo os padrões do Ministério da Saúde ${ }^{6}$, ambos os grupos de pacientes residentes em municípios com média e alta endemicidade, independente do modo de detecção, apresentaram a alta proporção de casos com idade inferior a 15 anos. Somando-se a isto, outro fator importante foi a alta proporção de pacientes de ambos os municípios com grau de incapacidade igual a 2 no início do tratamento, confirmando assim o diagnóstico tardio dos casos. Estes achados mostraram-se preocupantes, pois, a detecção em menores de 15 anos é o principal indicador de monitoramento da endemia, porque permite detectar focos de transmissão ativa da doença podendo sinalizar exposição precoce e manutenção da cadeia de transmissão ${ }^{8}$. Ainda levando-se em consideração o grande percentual de pacientes de ambos municípios detectados com grau de incapacidade física já instalada, e sabendo-se que a incapacidade física está relacionado com o tempo de doença, pode-se considerar os resultados como evidência de diagnóstico tardio nestes pacientes. Estes indicadores permitem uma avaliação indireta da efetividade das atividades de detecção precoce de casos e da prevalência oculta ${ }^{9}$.

Com relação ao grau de incapacidade física ao final do tratamento, a análise utilizando a regressão logística, demonstrou que o grupo de pacientes provenientes do município de Duque de Caxias apresentavam uma proporção significativamente maior de pacientes com grau de incapacidade física igual a 2 quando comparados àqueles residentes no município do Rio de Janeiro. Este achado não esteve relacionado à maior presença de quadros reacionais no momento do diagnóstico. As condições socioeconômicas e a baixa escolaridade associada à demora na procura pelo diagnóstico podem ter contribuído para esta maior proporção de casos entre os pacientes residentes em Duque de Caxias.

Quanto ao índice baciloscópico inicial ter se mostrado mais elevado entre os pacientes de Duque de Caxias, indicando uma condição de menor precocidade no diagnóstico desses casos, não foram observadas diferenças para o índice baciloscópico final, indicando a efetividade no tratamento.

Vários estudos demonstram que a hanseníase é fortemente relacionada com as condições de vida e pobreza ${ }^{10,11}$, e segundo Cunha et al. ${ }^{5}$, evidencia-se uma tendência de concentração dos doentes em camadas da sociedade menos favorecidas, corroborando com os achados do presente estudo que observou menor nível de escolaridade e menor renda familiar entre os pacientes residentes no município de Duque de Caxias do que no Rio de Janeiro, nos dois últimos períodos. Enquanto que entre os pacientes residentes no Rio de Janeiro a média se manteve similar entre os períodos, entre os pacientes residentes em Duque de Caxias, observou-se uma importante queda na renda média familiar dos pacientes.

Por se tratar de um serviço de referência nacional, a casuística analisada no presente estudo é particularmente sujeita a determinadas especificidades (como casos de difícil diagnóstico, maior gravidade da doença, etc.) e, portanto, vícios evidentes de seleção, esses dados não correspondem a uma amostra representativa de todos os casos de hanseníase da população base de onde a clientela do ASA é proveniente, mas constituem uma casuística cujo atendimento é claramente diferenciado daquele habitualmente oferecido na rede de unidade de saúde do SUS. Por outro lado, o atendimento em um centro de referência está menos sujeito a problemas operacionais e a despadronização de procedimentos e profissionais. Os achados de um serviço de referência contribuem para evidenciar tendências recentes da epidemia nos seus desdobramentos mais graves e preocupantes, e nortear ações de controle e de prevenção da doença. Os presentes achados permitem confirmar a necessidade de atividades mais efetivas no controle da hanseníase nos dois municípios estudados.

Os achados do presente estudo corroboram os de outros que abordam as diferenças referentes a diversos agravos em saúde entre diferentes cidades sugerindo que fatores inter e intraurbanos (por exemplo, segregação residencial) desempenham um importante papel na saúde coletiva, 
indicando que é a multiplicidade e a interação de fatores em diferentes níveis que determinam a saúde das populações urbanas ${ }^{12}$.

Cliff et al. ${ }^{13}$ mostram que, para diferentes doenças infecciosas com longo período de incubação, a distribuição se mostra distinta em função das características geográficas e econômicas das cidades, e que quanto maior o período de incubação da doença maior a variação da forma da distribuição das doenças de uma cidade para outra.

Os presentes dados sugerem uma condição de endemicidade mais acentuada no município de Duque de Caxias do que no do Rio de Janeiro em um passado não muito distante. Porém os mais recentes apontam para uma reversão deste quadro epidemiológico ${ }^{13}$. Em trabalho recente, que analisou a situação epidemiológica do município de Duque de Caxias, foi verificado que ações de descentralização da assistência, possibilitaram um incremento da deteç̧ão de casos novos e favoreceu a precocidade do diagnóstico. No entanto, as diferenças regionais também devem ser consideradas, pois estão relacionadas com a endemicidade da área ${ }^{5}$.

O município de Duque de Caxias apresenta indicadores socioeconômicos menos favoráveis do que o Município do Rio de Janeiro, no que diz respeito à condição familiar, condição de moradia e desenvolvimento humano ${ }^{7}$. Tais condições poderiam estar relacionadas, em parte, considerando as limitações de conclusões ao nível ecológico, as diferenças encontradas no presente estudo entre os pacientes residentes em ambos os municípios que foram acompanhados em um mesmo centro de referência, sugerindo a existência de influência de determinantes sociais na saúde dos indivíduos. Por outro lado, conforme observado neste estudo, a oferta de tratamento e de acompanhamento diferenciado pode favorecer a prevenção de incapacidades e o controle do índice baciloscópico, desfechos estes que poderiam apresentar resultados desfavoráveis em razão de fatores sociais e econômicos ao nível individual e ao nível populacional.

No presente estudo ainda observou-se que a proporção de abandono de tratamento foi baixa e a regularidade do tratamento foi elevada para os pacientes de ambos os municípios, revelando proporções similares, independente da origem do paciente, sugerindo que a distância da residência à unidade de saúde parece não afetar a adesão ao tratamento. Estes resultados também sugerem que o tratamento oferecido de forma satisfatória contribui em minimizar o efeito que os fatores contextuais podem apresentar sobre os desfechos de saúde.

Embora o desenvolvimento de cada doença seja um fenômeno biológico individual, é possível que para diversas, senão todas as doenças, estes determinantes não possam ser inteiramente operacionalizados apenas ao nível individual ${ }^{14}$. Em se tratando de doenças infecciosas, a dimensão ecológica é indispensável, pois a determinação é, inevitavelmente, individual e coletiva ${ }^{15}$. Para isso, técnicas estatísticas apropriadas devem ser empregadas, como a modelagem multinível, que considera diferentes níveis hierárquicos de variáveis que se referem a fatores individuais e ecológicos.

\section{Colaboradores}

MAVB Hacker realizou as análises estatísticas, interpretou os resultados e redigiu o artigo. AM Sales, ECA Albuquerque, E Rangel e JAC Nery participaram da interpretação dos resultados. NC Duppre participou da interpretação dos resultados e revisão critica do texto EN Sarno foi responsável pela concepção e interpretação dos resultados. 


\section{Referências}

1. World Health Organization (WHO). Weekly epidemiological Record. 2010; 35(85):337-348.

2. Brasil. Ministério da Saúde (MS). Vigilância em Saúde situação epidemiológica da hanseníase no Brasil [site na Internet]. Brasília: Programa Nacional de Controle de Hanseníase. [acessado 2010 ago 13]. Disponível em: http://portal.saude.gov.br/portal/arquivos/ pdf/boletim_novembro.pdf.

3. Boerma JT, Weir SS. Integrating demographic and epidemiological approaches to research on HIV/ AIDS: the proximate-determinants framework. $J$ Infect Dis 2005; 191(Supl. 1):S61-67.

4. Adimora AA, Schoenbach VJ. Social context, sexual networks, and racial disparities in rates of sexually transmitted infections. J Infect Dis 2005; 191(Supl. 1):115-122.

5. Cunha MD, Cavaliere FA, Hércules FM, Duraes SM, de Oliveira ML, de Matos HJ. The impact of leprosy elimination strategy on an endemic municipality in Rio de Janeiro State, Brazil. Cad Saude Publica 2007; 23(5):1187-1197.

6. Brasil. Dados e Indicadores Selecionados [site na Internet]. Brasília: Secretaria de Vigilância em Saúde. Departamento de Vigilância Epidemiológica. [acessado 2010 abr 15]. Disponível em: http:// portal.saude.gov.br/portal/arquivos/pdf/caderno_ de_indicadores_hanseniase_brasil2001a2008.pdf

7. Organização das Nações Unidas (ONU). PNUD. Atlas do Desenvolvimento Humano. [site na Internet] Salvador: ONU. [Acessado em 2010 out 25]. Disponível em: http://www.pnud.org.br.

8. Brasil. Ministério da Saúde (MS). Sistema de Vigilância Sanitária. Brasil reduz casos de hanseníase em menores de 15 anos. Edição 69 - janeiro de 2010. [site na Internet]. Brasília: MS. [acessado 2010 nov 18]. Disponível em: http://189.28.128.179:8080/ svs_informa/edicao-69-janeiro-de-2010/brasil-reduz -casos-de-hanseniase-em-menores-de-15-anos.
9. Brasil. Ministério da Saúde (MS). Plano Nacional de Eliminação da hanseníase em nível Municipal 20062010. [site na Internet]. Brasília: Secretaria De Vigilância Em Saúde. Departamento De Vigilância Epidemiológica. Programa Nacional De Eliminação Da Hanseníase [acessado 2009 mar 17]. Disponível em: http://bvsms.saude.gov.br/bvs/publicacoes/ hanseniase_plano.pdf.

10. Duarte MT, Ayres JA, Simonetti JP. Socioeconomic and demographic profile of leprosy carriers attended in nursing consultations. Rev Lat Am Enfermagem 2007; 15(No Esp.):774-779.

11. Helene LM, Salum MJ. Social reproduction of leprosy: a study of patients profile with leprosy in the city of São Paulo. Cad Saude Publica 2002; 18(1):101113.

12. Gálea S, Freudenberg N, Vlahov D. Cities and population health. Soc Sci Med 2005; 60(5):1017-1033.

13. Cliff AD, Hagget P, Raynor MS. The global sample: an overall picture, In: Cliff A, Haggett P, SmallmanRaynor M, organizadores. Deciphering global epidemics: analytical approaches to the disease records of world cities. New York: Cambridge University Press; 1998. p. 450.

14. Diez-Roux AV. Bringing context back into epidemiology: variables and fallacies in multilevel analysis. Am J Public Health 1998; 88(2):216-222.

15. Halloran ME, Struchiner CJ. Causal inference in infectious diseases. Epidemiology 1995; 6(2):142-151.

Artigo apresentado em 29/04/2011

Aprovado em 05/05/2011

Versão final apresentada em 01/09/2011 